\title{
A Survey-based Study of Grid Traffic
}

\author{
Yehia El khatib, Christopher Edwards \\ Computing Department \\ InfoLab21, South Drive \\ Lancaster University \\ Lancaster, LA1 4WA, UK \\ \{yehia,ce\}@ comp.lancs.ac.uk
}

\begin{abstract}
Grid computing offers the prospect of harnessing huge amounts of computational resources. However, it is being argued that such potential cannot be fully exploited due to the nature of the Internet architecture which is not suitable for high-speed communication of large data volumes. In an attempt to find out the accuracy of such description of grid application traffic, we have conducted a survey of Grid applications. Among other things, the survey results have revealed the diversity of Grid traffic, suggesting that there is more to Grid traffic than just transfers of huge bulks. In this paper, we present these results and identify different trends of traffic behavior that have been observed within the results. We then validate our findings by looking in detail at two of the applications that we have surveyed.
\end{abstract}

\section{Categories and Subject Descriptors}

C.2.1 [Computer-Communication Networks]: Network Architecture and Design - Distributed networks; C.2.4 [ComputerCommunication Networks]: Distributed Systems - Distributed applications.

\section{INTRODUCTION}

Grid computing offers the prospect of gaining huge amounts of computational resources for a fraction of the cost that would be paid to actually own such resources. It is a safe investment for any organization whose work involves computations that require abundant resources, such as processing power, storage space, etc. Grid computing, therefore, enables organizations to harness the most out of their resources regardless of how geographically scattered or locally administrated these resources are.

However, it is being argued that present-day networking technologies are not suitable for the kind of traffic that is transmitted in Grids [21]. In particular, it is suggested that the limitations of the TCP/IP stack prevent Grids from working to their full potential $[2,7,19]$. However, this argument is largely based on the assumption that Grid traffic is mostly large bulks of data [13, $15,19]$. While there is indeed sufficient evidence that TCP is not suitable for high-speed bulky data transfers $[2,3,9,10,11,12,18$, 20], there is not much to support the assumption that large bulks of data dominate the traffic of Grid applications. We believe that this

GridNets 2007 October 17-19, 2007, Lyon, France. Copyright 2007 ICST ISBN 978-963-9799-07-3.

DOI 10.4108/gridnets.2007.2120 assumption has been based on speculations and forecasts of how Grid applications work.

The argument that current Internet technologies are inept for Grid communications provided the inspiration behind EC-GIN (EuropeChina Grid InterNetworking) [23], a European-funded research project for improving the ability of the network to support Grid applications. One of the objectives of EC-GIN is to introduce a comprehensive networking API, called GinTonic, that provides new programming abstractions designed to improve the performance of network communication across the Grid. For the architectural design of GinTonic, understanding the requirements of Grid applications is crucial. It might be easy enough to predict these requirements according to our perception of Grid applications. However, a close look at some applications that are currently in operation would yield a more realistic set of requirements.

This has motivated us to conduct a survey of current Grid applications, the results of which will guide the architectural choices in the design of GinTonic. In this survey, we look at different characteristics of the Grid applications, their middleware environments, their traffic footprints, and most importantly their network requirements. The survey also presents us with evidence that Grid traffic is not necessarily "mice and elephants" [17], i.e. a combination of very small control signals and very large bulks of data transfer.

The rest of this paper is arranged as follows. Section 2 describes the aim and process of conducting the survey, while the results are summarised in section 3 . In section 4 , we identify five distinct trends in the traffic of the surveyed Grid applications. We then, in section 5, discuss two of the surveyed applications to show how their traffic footprints originate from their behaviours. Section 6 offers some concluding remarks and, finally, section 7 presents future work.

\section{SURVEY OF APPLICATION REQUIREMENTS}

This section gives an overview of the conducted survey. We discuss the aim and process of conducting the survey.

\subsection{Aim}

The aim of the survey is to draw a clearer picture of what the network requirements of Grid applications are, based on the specifications of deployed applications. The results give a recommendation of the services that need to be included in the API design. The results also describe some aspects of the applications such as scale, composition, dataset granularity, delay-sensitivity, middleware, accounting metrics, etc. The output of the survey, however, is not intended to be a comprehensive statistical analysis 
of the different aspects of Grid applications.

\subsection{Target Audience}

A questionnaire was sent out to a number of projects which employ or are in the process of developing Grid applications. Due to the technical nature of some of the questions, we intentionally targeted people who have adequate experience with Grid applications. This includes the developers, administrators, and advanced users who have used the system enough to know about its behaviour and requirements. In a small number of cases, participants were asked for a short face-to-face interview to obtain more details or to clarify their responses.

\subsection{Contents}

The questionnaire was kept as simple as possible, considering that it was not created for the purpose of conducting a thorough investigation of Grid application details. The questionnaire is made up of two pages plus an introductory front-page, and it consists of a collection of multiple-choice questions as well as a final openended question. However, it is noteworthy that even the multiplechoice questions in the survey leave room for the participants to write further comments, considerations or supporting statements. The participants were asked to answer to the best of their knowledge, using approximations for any numerical values.

The questionnaire was divided into the following 12 sections:

- Description of the Grid Application: In this section, the participants are asked to give a brief overview of the application and its purpose.

- Scale: This section enquires about the scale of the Grid used, in terms of nodes and administration domains.

- Composition: The participants are asked about the device diversity in their Grid. This aims at getting a feel for the type of computational resources available in the Grid.

- Data Granularity: Grid traffic is commonly referred to as being dominantly made up of large bulks of data $[13,15,19,21]$. However, in order to reach efficient communication, we need to know more about those "bulks of data" and about the traffic in general. This is where the importance of this section arises. Here, the participants are asked to provide the amounts and pattern of the traffic.

- Data Timeliness: Similar to the previous section, this section intends to find out more about the characteristics of the traffic in Grid networks. In this case, the quest is to learn about any delayintolerant traffic and the reason behind such delay-sensitivity.

- Encryption: This section asks about the amount of encrypted traffic and about the provisioning of encryption services.

- Accounting: In this section, we ask the participant about the metrics their application considers for accounting.

- Data Replication: This section addresses the topic of replica management which Grids utilise in order to optimise reliability and efficiency. We will use the results from this section to deduce whether or not GinTonic is required (or rather expected) to facilitate such functionality.

- Data Path: Here we enquire about one-to-many communication services used by the application. Again, this is a service that we might want GinTonic to provide.

- Network Transport Protocol: The aim of this section is to learn about the importance of connection-oriented communication as opposed to connectionless-oriented communication in the context of Grid applications.

- Middleware: Here we ask about the middleware solutions employed.

- Special Network Services: This section enquires about additional network services required by the application.

\section{RESULTS OF THE SURVEY}

In this section, we present the results obtained from analysing the collected set of data from the survey described in the previous section.

\subsection{Use}

As illustrated in Figure 1, 18\% of the applications we surveyed are used for Particle Physics research; 13\% are used for Astronomy; $13 \%$ for Engineering; $13 \%$ for some form of mathematical analysis computations; $13 \%$ for Social Sciences; while the remaining 30\% were equally used for the following: Environmental Sciences, Medicine, Meteorology, software development, visualization.

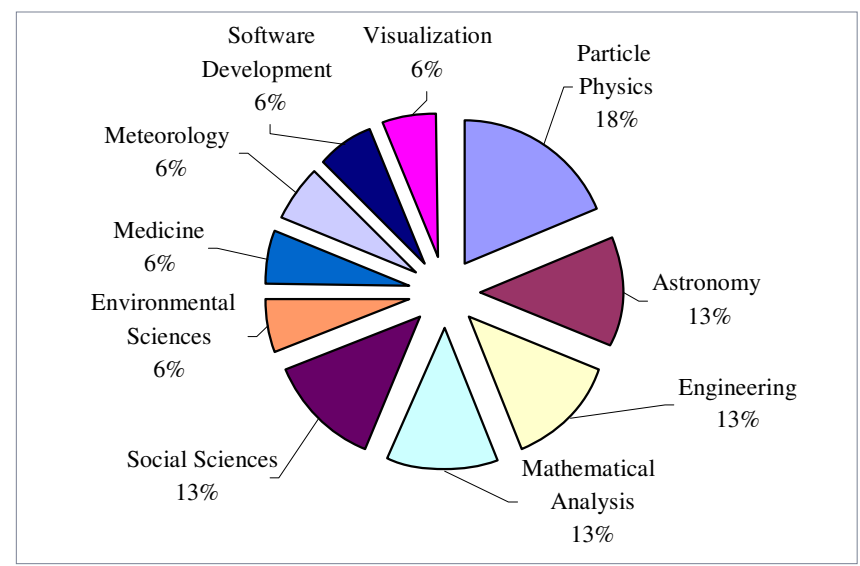

Figure 1. The research fields of the surveyed applications

\subsection{Scale}

Of the surveyed applications, $13 \%$ are deployed over Grids made up of 1-10 nodes, $54 \%$ are deployed over $100-400$ nodes, $20 \%$ are deployed over 400-1000 nodes, and 13\% are deployed over Grids of more than 1000 nodes. This is shown in Figure 2.

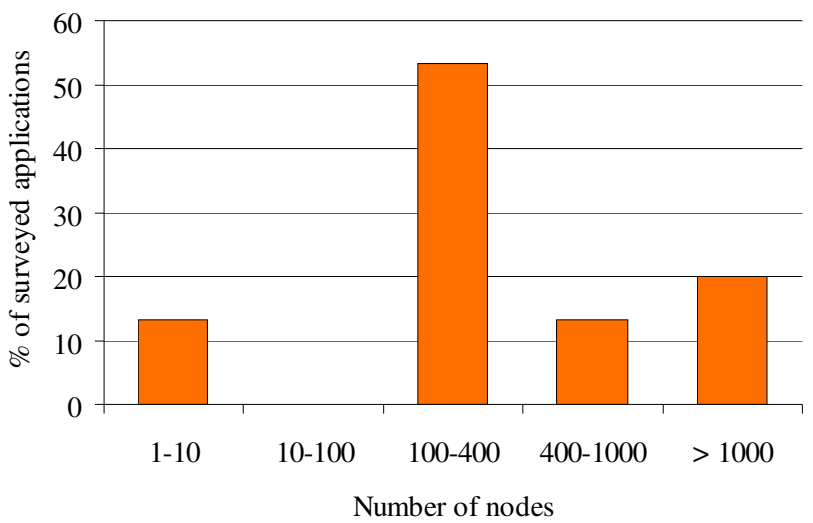

Figure 2. The scale of the applications in number of nodes 
The majority of these Grids (71\%) span across 1-10 administrative domains, while $21 \%$ have nodes in 10-100 different domains. Only $8 \%$ of the surveyed applications are deployed over a Grid that has nodes in more than 1000 different domains. Figure 3 portrays these statistics.

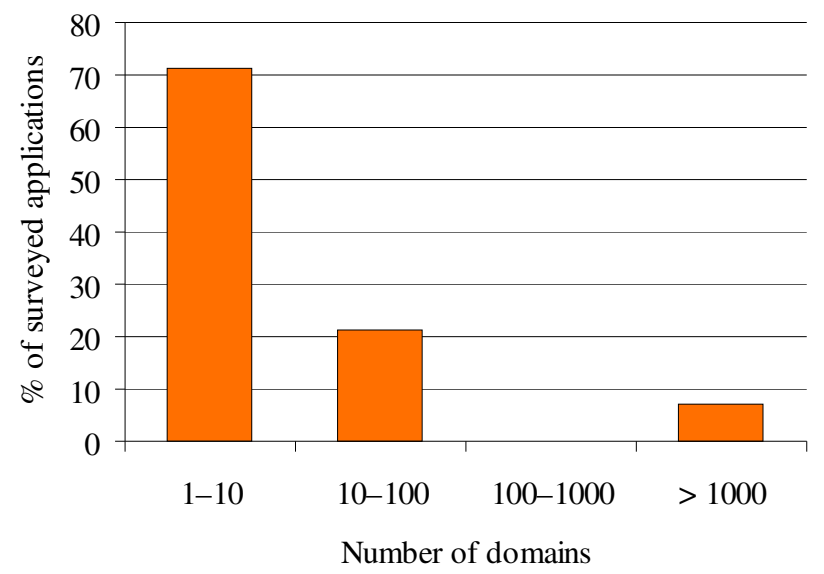

Figure 3. The scale of the applications in number of administrative domains

\subsection{Composition}

$47 \%$ of the surveyed applications are deployed solely on dedicated clusters. Only $7 \%$ of the surveyed applications are deployed on a Grid free of clusters, consisting only of desktop computers. The remaining Grids (46\%) are almost equally composed of clusters and desktop machines.

It is worth noting that only one application uses small devices (such as embedded processors) and they only constitute less than $1 \%$ of the total number of devices in that Grid. In addition, there are no applications that have mobile phones as nodes in their Grid. The overall composition ratio is illustrated in Figure 4.

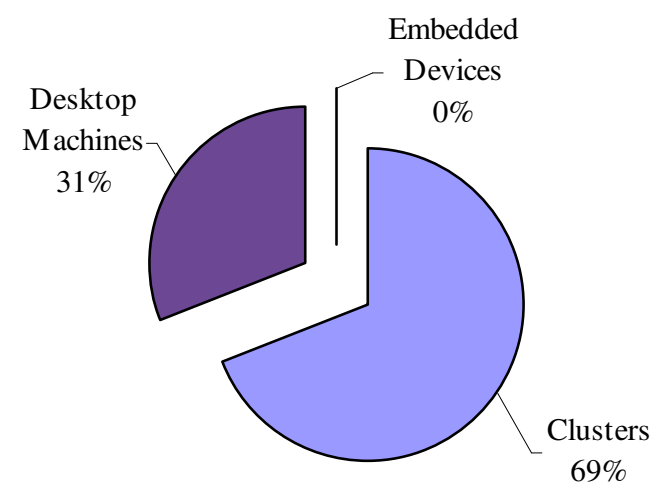

Figure 4. The overall grid compositions

Further inspection revealed some relationships between certain application types and the composition of their Grids. All the surveyed applications that are involved in image analysis are deployed on Grids composed solely of dedicated clusters. All surveyed simulation applications are deployed on almost $100 \%$ clusters, while all surveyed data management applications are deployed over Grids where there are far more desktop computers than dedicated clusters.

\subsection{Dataset Granularity}

Based on the values given by the participants, the survey revealed that the three most common dataset sizes are $10 \mathrm{kB}, 10 \mathrm{MB}$, and $100 \mathrm{~GB}$. These are visible as the peaks in Figure 5, which depicts the logistic distribution of dataset sizes. Figure 6 illustrates the corresponding sigmoid curve. The $\mathrm{x}$-axes in both figures are to logarithmic scale.

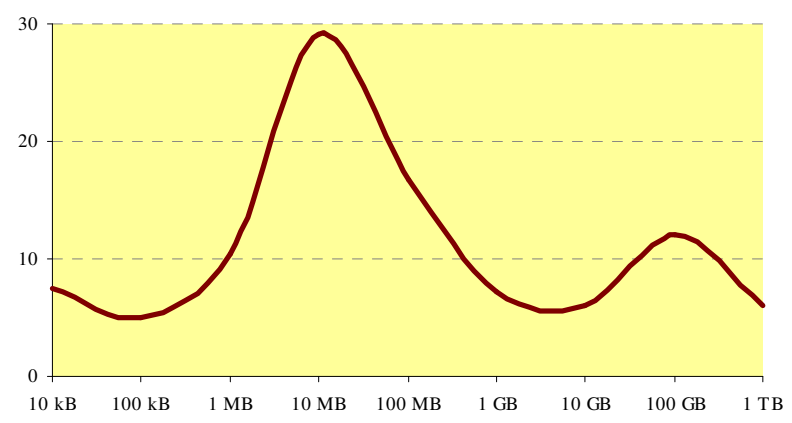

Figure 5. The probability density function of dataset sizes

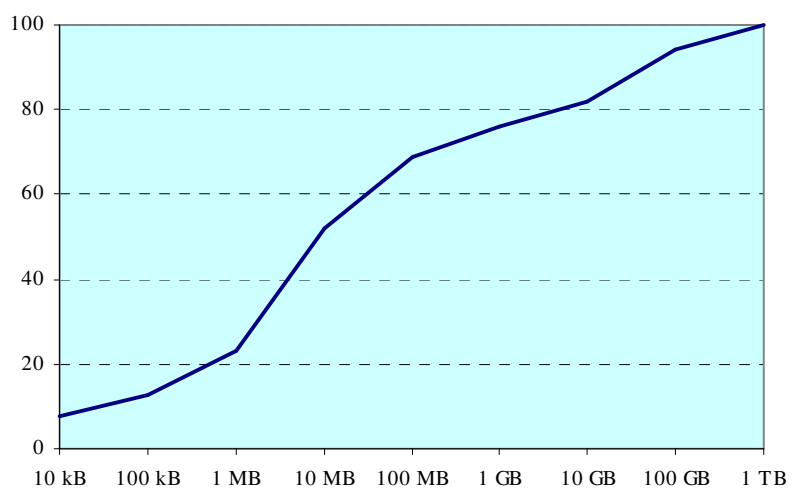

Figure 6. The cumulative distribution function of dataset sizes

A closer look at the numbers reveals that almost $12 \%$ of the datasets of all surveyed applications are in bulks smaller than $100 \mathrm{kB}$ in size, $55 \%$ are in bulks of $1-100 \mathrm{MB}$, and $18 \%$ are in bulks of $10 \mathrm{~GB}$ or more.

Although only to a limited extent, these numbers show how different Grid traffic is when compared to generic IP traffic (such as Web traffic). Moreover, they illustrate how mixed the dataset sizes are. Dataset sizes range from a few kilobytes in size to megabytes, hundreds of megabytes, gigabytes, hundreds of gigabytes, and could in a few cases stretch even more. The coexistence of such diverse traffic sizes affects the transmission performance of all traffic [17].

This observation is equally important because it refutes the common belief that Grid traffic consists predominantly of large bulks, as the results clearly demonstrate that the majority of dataset sizes are below the gigabyte limit.

\subsection{Data Timeliness}

Certain applications are time-critical and they need to enforce deadlines on the delivery of their packets. Packets that arrive later than the deadlines are considered of no use and are discarded. Embarrassingly parallel applications, on the other hand, do not typically impose such deadlines.

One of the applications we surveyed is being used for forecasting 
Alpine watersheds and thunderstorms based on parameter measurements from data collection points deployed in the field. Data that arrives late has to be discarded in order to process the data that is due. Besides this application, only one more of the surveyed applications imposes a deadline on the delivery of non-multimediastream data packets. Although this latter application involves Web service invocations which are asynchronous by nature, the application imposes strict deadlines on the delivery of Web service results. This reflects the essence of promptness in this application.

Interestingly, the part of the data that is time-sensitive in the two applications discussed above is mainly the part that is transferred in bulks in the order of tens of megabytes.

\subsection{Encryption}

Although security is a major concern of Grid applications [4], only $44 \%$ of the surveyed applications encrypt their data prior to sending it over the network. Of these applications, 57\% rely on the middleware to provide the encryption as opposed to encryption being carried out entirely by the network transport layer.

It was observed that all the surveyed Particle Physics applications delegate the middleware to encrypt $100 \%$ of their data, while all the surveyed Social Sciences applications rely on the network transport layer protocol for the same purpose.

\subsection{Accounting}

When asked about the metrics considered important for accounting measures, the great majority $(94 \%)$ of the participants chose CPU processing power, $63 \%$ chose disk storage space, and 38\% chose network bandwidth utilization. Some participants mentioned more specific accounting factors such as service invocations, number of employed CPUs, code generation number, and software licenses.

\subsection{Data Replication}

$44 \%$ of all surveyed applications replicate at least a portion of the data they push into the network. It was noticed that all surveyed Particle Physics applications replicate all their data.

\subsection{Data Path}

The created traffic that has more than one recipient amounts to $22 \%$ of the total traffic of all surveyed applications.

Only $44 \%$ of the surveyed applications employ one-to-many communication schemes. These applications all use multicast one way or another. Two thirds of these applications integrate a multicasting mechanism into their code, while the other third employs middleware multicasting services.

Besides multicast, only one application uses an anycast scheme [16], which is provided by the middleware. The same application also implements its own means of scavenging, an advanced anycasting scheme where the recipients of the data are chosen according to specific criteria set forth by the application and verified by the resource brokering element of the middleware.

\subsection{Network Transport Layer}

All surveyed applications utilise TCP as the network transport protocol. This shows how important reliable communication is to Grid applications.

One of the applications also uses UDT (UDP-based Data Transfer
Protocol), which is an application-level transport protocol designed for high-speed WANs [7], for testing purposes only.

\subsection{Middleware}

$44 \%$ of the surveyed applications run on top of a Globus-based grid computing environment. Other applications use EGEE-LCG (19\%), Askalon (11\%), Condor (4\%), DIET (4\%), jBoss (4\%), OAR \& SCP $(4 \%)$, or Unicore $(4 \%)$. Only one surveyed application uses a proprietary middleware solution specifically created for the project.

One of the unanticipated facts that the survey has revealed is that $56 \%$ of all applications run on top of more than one middleware solution. Some $44 \%$ of the applications use exactly two middleware solutions, all of them having Globus as one of them; $19 \%$ of the applications employ Globus along with Askalon, while another 13\% use Globus with EGEE-LCG. Furthermore, $12 \%$ of the applications use three middleware solutions, all of which included EGEE-LCG as one of the three.

\subsection{Special Network Services}

Although $31 \%$ of the participants were not aware of any transfer delay prediction services, most of them were. Indeed, 54\% agreed that it would be very useful for their applications, only $8 \%$ were already using such services, while another $8 \%$ thought it was unnecessary.

As for advanced network reservation, only $15 \%$ of the applications employ it to ensure the quality of communication before it commences, whereas $23 \%$ think that it would be useful for their applications but they do not currently employ it. Also, another $23 \%$ ruled out the use of advanced reservation completely and $38 \%$ were unsure about the vitality of such services.

The provisioning of network topology information appears to be a new concept to almost $54 \%$ of the survey participants. About $15 \%$ of the participants do not think that network topology information would be of any use to their respective applications. However, $31 \%$ of them said that their Grid application would most definitely make use of such service if available. These participants mentioned that knowledge obtained from such a service would help make data transfer operations more efficient. One participant said that the application his institution developed involves various operations where the knowledge of the closest node to any given node would be very useful. Another participant said that knowledge of the network topology would help their scheduler in distributing work in a more efficient manner.

Two of the survey participants mentioned that their Grid applications require extra resource brokering on top of that provided by the middleware. One of these stated that they use additional resource brokering software on top of Globus just to manage shared memory across Grid nodes. The other said that the application they developed needs more information about CPU usage and availability across the Grid. This latter application runs over a proprietary middleware solution.

One thing all participants seemed to agree on is that an API such as GinTonic should maintain the ease of secure communication across multiple administration domains. The necessity of secure communication is an obvious requirement of the API and perhaps any similar integrated connectivity interface. In Grids, it is necessary to maintain interoperability at different levels across all domains and thus achieving security is made more difficult [4]. 
According to the participants, achieving secure communication using any of the current middleware solutions is a cumbersome task.

Lastly, almost all participants agreed that special network-related operating system services and special networking hardware are not required to run their applications.

\section{ANALYSIS OF TRAFFIC BEHAVIOUR}

The survey results (section 3.4) indicate the non-conformance of Grid traffic to common belief. In order to emphasize this contrast and highlight the different ways Grid applications utilise the network, we classify the surveyed applications according to their traffic behaviour as identified by the sizes of their datasets.

From the survey results, we noted five main classes of traffic behaviour. We list these different classes below and comment on the common aspects observed within the applications of each class. Figure 7 displays the range of average dataset sizes for each of the different classes. The $\mathrm{x}$-axis in Figure 7 is to logarithmic scale.

The aim of this effort is not to typecast Grid applications. Our aim is to distinguish the magnitude of difference in traffic behaviour and to investigate the causes of such differences. Moreover, the recognition of such differences will help in the design of a more realistic network-enhancing API.

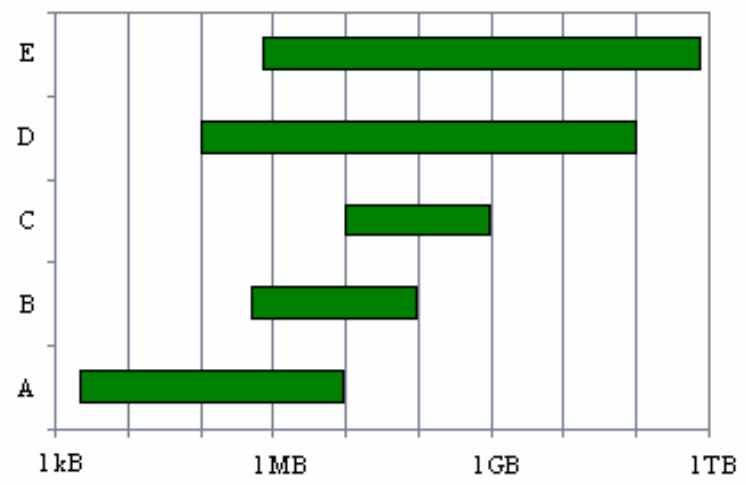

Figure 7. Average dataset sizes of the different traffic behaviour classes

\subsection{Class A}

The first class of traffic behaviour is the most noticeable due to the fact that although it might be the least anticipated behaviour of Grid applications, $34 \%$ of all the surveyed applications fall under this class. This includes applications that deal mostly with lightweight datasets; ones which are never larger than 5-10 MB. These applications are used for either mathematical calculations or distributed data management in projects related to mathematical analysis, Engineering, and Social Sciences.

\subsection{Class B}

Applications which can be grouped under this class have datasets between 0.5 and $100 \mathrm{MB}$ in size. Moreover, the variance in dataset sizes in this class is relatively large, i.e. dataset sizes of each application under this class tend to be at both ends of the mentioned range. Of all surveyed applications, $20 \%$ fell under this class. These included image analysis as well as simulation applications. Remarkably, all applications in this class are used for either
Astronomy or Meteorology. Furthermore, all these applications were deployed over Grids of 100-300 nodes across 6-8 administrative domains.

\subsection{Class C}

$13 \%$ of the surveyed applications had all their dataset sizes in the relatively narrow range of $10 \mathrm{MB}-1 \mathrm{~GB}$. These applications are used for advanced software development techniques and distributed data management. All applications in this class are deployed on Grids that are made up mostly of desktop machines, making this class the only one with a vivid relationship between the composition of the Grid and the traffic behaviour.

\subsection{Class D}

The fourth class contains applications whose dataset sizes vary within a wide range from $100 \mathrm{kB}$ to $100 \mathrm{~GB}$. However, the majority of the datasets are between $10 \mathrm{MB}$ and $10 \mathrm{~GB}$ in size. $13 \%$ of the surveyed applications fall under this class, and they are used for simulations, mathematical modeling, calibrations and complex computations.

\subsection{Class E}

The fifth and final class contains the heavyweight applications that have received the most attention in Grid computing literature. The main focus of these applications is the analysis of very large datasets, in the order of tens to hundreds of gigabytes, as well as other datasets as small as a few megabytes in size. The $20 \%$ of our survey population that fall under this class are being used for Particle Physics, Engineering and Social Sciences in order to perform complex numerical analysis and/or large-scale simulations. These applications run over huge Grids made up of thousands of nodes, including clusters, desktop machines, and small devices (such as embedded processors), spanning across a large number of administrative domains.

\subsection{Discussion of the Classification}

With the exception of class $\mathrm{C}$, there seems to be no clear relationship between the average size of the datasets and the composition of the Grid. There are applications that handle datasets of sizes in the order of gigabytes (such as class D or E applications) and there are others which have the majority of the datasets in the order of a few kilobytes (such as class A). Nonetheless, there is enough evidence in the survey results to suggest that the all applications are capable of running on Grid systems that are entirely made up of clusters or desktop machines. Furthermore, there is no solid association, other than the one observed in class B, between the research field for which the application is used and the amount or pattern of traffic it creates.

It is interesting that the ranges of dataset sizes of classes D and E are much wider than those of the other classes. This can be easily discerned from Figure 7. Perhaps this is due to the fact that applications in classes D and $\mathrm{E}$ are used for intensive computations, such as simulations and complex numerical processing, which, according to the evidence presented by the survey, require the transfer of very large bulks of data as well as a significant amount of small datasets.

Our survey included applications in various fields of scientific research including Particle Physics, Meteorology, Astronomy, Engineering, mathematical analysis, Social Sciences, and Medicine. 
However, they do not all involve the transfer of huge data volumes. In fact, the most common class of traffic behaviour is class A (see Figure 8) which involves datasets no larger than $10 \mathrm{MB}$.

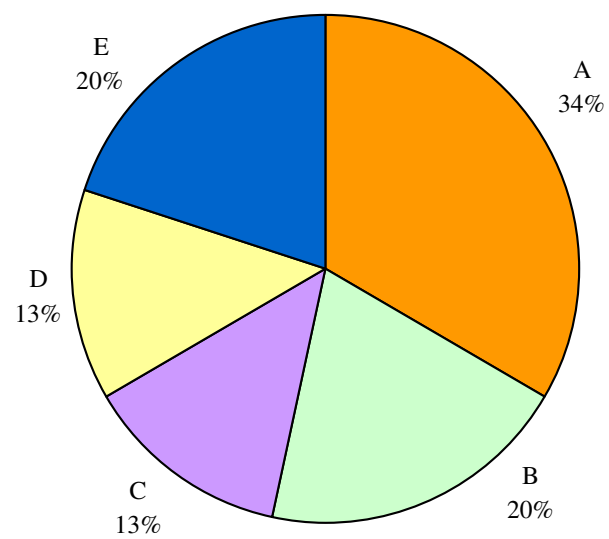

Figure 8. The distribution of the traffic behaviour classes amongst the surveyed applications

\section{EXAMPLES OF SURVEYED APPLICATIONS}

In order to show how the purpose and nature of a grid application affect its traffic behaviour, we report in some detail on two of the applications that have been included in the survey and apply the classification scheme to them. First, we give an outline of the purpose of each application, how it works and its typical traffic pattern. Then, in view of that, we place each application under one of the aforementioned classes.

\subsection{ATLAS for LHC}

LHC, or the Large Hadron Collider [26], is a particle accelerator and collider and is also the world's largest machine, costing a total of $£ 2.6$ billion. Located at CERN near the Switzerland-France border, LHC will see its first particle collisions by November 2007. The experiment is planned to run for nine consecutive months and then cease for three months before commencing again. During its first active period, LHC is expected to trigger huge amounts of raw data in the neighbourhood of 10 petabytes. This harvested data will then be processed by the Grid and the results obtained will be compared to those of simulated experiments. ATLAS (A Toroidal LHC ApparatuS) [22] is one of the five particle detector experiments that will run at LHC, and it is "the largest volume detector ever constructed for Particle Physics" [29]. ATLAS brings together almost 2000 scientists from around the world.

The Grid infrastructure for this project, the LHC Computing Grid (LCG) Project [27], is a collaboration between 165 scientific organisations, universities and government bodies connected together using a dedicated 10Gbps lightpath. These sites are organised using a three-tier distribution architecture. Tier 0 is the Particle Physics laboratory at CERN where part of the data analysis will take place. However, all Particle Physics aside, the main function of the laboratory is to farm out the raw data over the Grid to the Tier 1 sites.

There are ten Tier 1 sites scattered across France, Germany, Italy, Japan, the Netherlands, the UK and the US. Each of these sites has a cloud of Tier 2 sites associated with it. Tier 1 sites are responsible for splitting up the raw data they receive (from Tier 0) between their respective Tier 2 sites. Each Tier 2 site processes the data upon receiving it, stores the results locally on magnetic disk, and sends a copy of the results to its respective Tier 1 site where it is stored on tape. Hence, there are always at least two copies of every result file in the Grid.

Most data is sent as chunks in the order of (a few) gigabytes at scheduled times during which sufficient network resources would have been reserved in advance. However, some datasets of special importance are transferred on demand regardless of the prescheduled times. Also, data processing results returned from Tier 2 to Tier 1 are sent whenever they are ready (ad hoc submission).

Data is stored in physical files of 1-4 gigabytes each. Such large file sizes are necessary in order to reduce the overhead on magnetic and tape storage devices, as a small number of large files are easier to manage and index than a large number of smaller files.

A dataset can be a collection of any sort of raw data or processed information. It is written only once and is then never modified nor deleted (except on rare occasions). A single dataset can be composed of one or more physical data files. To the physicists (i.e. the users), however, the physical partitioning is invisible; they deal only with datasets. In contrast, catalogues are transactional metadata collections that may be simultaneously modified by more than one user.

When a job is submitted to the system it is typically executed at the location where the required datasets reside, regardless of where the job was initiated. The aim, of course, is to minimize the overhead induced by moving large datasets across the network. This strategy relies on the over-provision of other computational resources such as processing power and memory. After a job is executed, a dataset of results is returned to the user. This dataset is reasonably small in comparison to those containing raw data.

Hence, the traffic created by ATLAS is mostly made up of datasets in the order of a few gigabytes (i.e. the raw data), but it also consists of smaller datasets in the order of a few megabytes (i.e. the job results). Accordingly, ATLAS is classified under class D.

\subsection{GROWL for GeSRM}

SABRE (Software for Analysis of Binary Recurrent Events) [28] is an application developed to process very large amounts of longitudinal data. Such data is typically made up of millions of observations per dataset with a large number of parameters associated with every dataset. SABRE employs fast numerical algorithms and schedules them to run over different nodes across the Grid in a parallel fashion.

GROWL (Grid Resources On a Workstation Library) [25] is a toolkit that facilitates the use of client-server legacy applications on the Grid by employing SABRE in order to submit jobs to the legacy server. GROWL, thus, enables any pre-built service to be run over the Grid without the need for any modification to the service. All that is needed is to build a thin client that translates the users' jobs into SABRE Web services to be sent to a GROWL server. In turn, this server will then translate these Web service invocations into calls that are recognizable by the legacy server which resides at the same site as the GROWL server. Such separation of server logic from the client application makes it easy and flexible to distribute more than one copy of a client-server application across a Grid.

GeSRM [24] is a research project intended to develop a method of spatial analysis known as GWR, or Geographically Weighted 
Regression, to run over the Grid. GeSRM employs GROWL to submit a large number of computational tasks over the Grid using Web services. This approach minimizes the client footprint on users' machines. Nevertheless, GeSRM transfers very large datasets of spatially dispersed data between clusters. It is not uncommon for these datasets to stretch to $100 \mathrm{~GB}$ or more in size. At the other end of the scale, Web service invocations are quite lightweight and, although they are usually smaller than $1 \mathrm{MB}$, they do constitute almost $30 \%$ of the transferred traffic. This is because a large number of user jobs might induce only little data processing on the clusters. Therefore, we classify the traffic created by GROWL in this instance under class E.

\section{CONCLUSION}

We conducted a survey in which we focused on network-related features of Grid applications. The survey presented information about the applications such as the network functionality, network demands, middleware interaction, etc. Such information has proved useful for the design of GinTonic, an API aimed at improving the network performance of Grid applications.

The output of the survey has also presented evidence that there is more to Grid traffic than very large data volumes. Based on the results, we have suggested a classification scheme that distinguishes different traffic footprints. This classification points out the diversity in Grid traffic; $34 \%$ of all the surveyed applications have datasets under $10 \mathrm{MB}$ in size, $54 \%$ of all surveyed applications have datasets under $100 \mathrm{MB}$ in size, and $74 \%$ of all surveyed applications have datasets under $1 \mathrm{~GB}$ in size.

With these numbers in mind, the survey fables the belief that the majority of Grid traffic is mainly made up of enormous volumes of data. Quite the opposite, the results demonstrate that Grid traffic comes in all shapes and sizes and that many Grid applications do not produce very large datasets at all.

\section{FUTURE WORK}

The survey offers an overview of the size and nature of traffic exchanged in Grids. The results illustrate how traffic differs from one application to another. However, the results do not show how the traffic of one application fluctuates from one time to another. In a further effort to study Grid traffic, we intend to monitor the traffic of a number of Grid applications. Through carrying out detailed analysis and mathematical modeling of the monitored traffic, we are hopeful of providing a realistic representation of Grid traffic that can then be used in Grid simulators. However, monitoring any distributed system, including Grids, is no simple task [1, 8, 14], as several factors must be taken into consideration.

\section{ACKNOWLEDGMENTS}

The authors thank the European Commission for funding EC-GIN (FP6-2005-IST-6-045256), the work under which this paper was completed. The authors are grateful to the participants of the survey for their significant contribution, as well as everyone within the EC-GIN consortium who helped in disseminating the questionnaire. The authors are also grateful to Dr. Roger Jones of LHC at CERN, Mr. Brian Davies of The Particle Physics Research Group at Lancaster University, Prof. Robert Crouchley of The Centre for eScience at Lancaster University, and Dr. Daniel Grose of The Centre for e-Science at Lancaster University.

\section{REFERENCES}

[1] "Grid Network Monitoring: Demonstration of Enhanced Monitoring Tools". Deliverable D7.2, EU Datagrid Document: WP7-D7.2-0110-4-1. April 2007.

[2] W. Feng, P. Tinnakornsrisuphap. "The Failure of TCP in HighPerformance Computational Grids". In proceedings of the 2000 ACM/IEEE conference on Supercomputing (SC2000), article no. 37, Dallas, Texas, USA, November 2000. ISBN: 0-7803-9802-5.

[3] S. Floyd. "HighSpeed TCP for Large Congestion Windows". Request for Comments: 3649. December 2003.

[4] I. Foster, C. Kesselman, G. Tsudik, S. Tuecke. "A Security Architecture for Computational Grids". In proceedings of the 5th ACM conference on Computer and Communications Security, pp: 83-92, San Francisco, California, USA, November 1998. ISBN: 1-58113-007-4.

[5] I. Foster, C. Kesselman, S. Tuecke. "The Anatomy of the Grid: Enabling Scalable Virtual Organizations". International Journal of Supercomputer Applications, vol. 15, no. 3, 2001.

[6] I. Foster, C. Kesselman (ed). "The Grid: Blueprint for a New Computing Infrastructure”. Second edition, Morgan Kaufmann Publishers, 2004. ISBN: 1-55860-933-4.

[7] Y. Gu, R. Grossman. "UDT: An Application Level Transport Protocol for Grid Computing". Second International Workshop on Protocols for Fast Long-Distance Networks (PFLDnet2004), Argonne, Illinois, USA, February 2004.

[8] D. Gunter, B. Tierney, K. Jackson, J. Lee, M. Stoufer. "Dynamic Monitoring of High-Performance Distributed Applications". In proceedings of the 11th IEEE Symposium on High Performance Distributed Computing (HPDC-11), Edinburgh, UK, July 2002.

[9] E. He, R. Kettimuthu, Y. Gu, S. Hegde, M. Welzl, P. VicatBlanc Primet, J. Leigh, C. Xiong. "Survey of Protocols and Mechanisms for Enhanced Transport over Long Fat Pipes". Global Grid Forum White paper Draft (work in progress), Data Transport Research Group, 2003. Available at: http://www-unix.mcs.anl.gov/ kettimut/Survey.pdf

[10] C. Jin, D. X. Wei, S. H. Low, J. Bunn, D. H. Choe, J. C. Doyle, H. Newman, S. Ravot, S. Singh, F. Paganini, G. Buhrmaster, R. L. A. Cottrell, O. Martin, W. Feng. "FAST TCP: From Theory to Experiments". IEEE Network, vol. 19, no. 1, pp. 4-11, January-February 2005.

[11] D. Katabi, M. Handley, C. Rohrs. "Congestion Control for High Bandwidth-Delay Product Networks". In proceedings of the ACM SIGCOMM 2002 Conference on Applications, Technologies, Architectures, and Protocols for Computer Communications, session: Congestion Control, pp: 89-102, Pittsburgh, Pennsylvania, USA, August 2002. ISBN: 1-58113-570-X.

[12] T. Kelly. "Scalable TCP: Improving Performance in Highspeed Wide Area Networks". ACM SIGCOMM Computer Communication Review, vol. 33, issue 2, column: Technical papers, pp: 83-91, 2003. ISSN: 0146-4833.

[13] T. Lavian, D. Hoang, J. Mambretti, S. Figueira, S. Naiksatam, N. Kaushik, M. Inder, R. Durairaj, D. Cutrell, S. Merrill, H. Cohen, P. Daspit, F. Travostino. "A Platform for Large-Scale Grid Data Service on Dynamic High-Performance Networks". First International Workshop on Networks for Grid Applications (GridNets2004), San Jose, California, USA, October 2004.

[14] C. Lee, J. Stepanek, R. Wolski, C. Kesselman, I. Foster. "A Network Performance Tool for Grid Environments". In 
proceedings of the 1999 ACM/IEEE Conference on Supercomputing (SC99), Portland, Oregon, USA, November 1999. ISBN: 1-58113-091-0.

[15] L. Paisley, J. Sventek. "Real-time Detection of Grid Bulk Transfer Traffic". In proceedings of the $10^{\text {th }}$ IEEE/IFIP Network Operations and Management Symposium (NOMS 2006), pp 66-72, Vancouver, Canada, April 2006. ISSN: 1542-1201, ISBN: 1-4244-0142-9.

[16] C. Partridge, T. Mendez, and W. Milliken. "Host Anycasting Service". Request for Comments: 1546. November 1993.

[17] F. Travostino, J. Mambretti, G. Karmous-Edwards (ed). "Grid Networks: Enabling Grids with Advanced Communication Technology". John Wiley \& Sons Ltd, 2006. ISBN: 0-470-01748-1.

[18] D. Wei, C. Jin, S. Low, S. Hegde. "FAST TCP: Motivation, Architecture, Algorithms, Performance". IEEE/ACM Transactions on Networking (TON), vol. 14, issue 6 (December 2006), pp: 1246-1259, 2006. ISSN: 1063-6692.

[19] E. Weigle, W. Feng. "A Case for TCP Vegas in HighPerformance Computational Grids”. In proceedings of the 9th IEEE International Symposium on High Performance Distributed Computing (HPDC'01), San Francisco, California, USA, August 2001.

[20] M. Welzl. "Traceable Congestion Control”. International Workshop on Internet Charging and QoS Technologies (ICQT 2002), Zürich, Switzerland, October 2002.
[21] M. Welzl, M. M. Yousaf. "Grid-Specific Network Enhancements: A Research Gap?". In proceedings of the IEEE/IFIP International Workshop on Autonomic Grid Networking and Management (AGNM'05), Barcelona, Spain, October 2005.

[22] The ATLAS (A Toroidal LHC ApparatuS) Experiment. http://atlasinfo.cern.ch

[23] Europe-China Grid InterNetworking (EC-GIN), a Framework 6 STREP project, Action Line: IST-2005-2.6.5.1.c Grids China, Project Reference (Contract Number): 045256. http://www.ec-gin.eu/

[24] Grid-enabled Spatial Regression Models (GeSRM), a small grant project funded by the National Centre for e-Social Science (NCeSS). http://www.ncess.ac.uk/research/sgp/gesrm/

[25] Grid Resources On a Workstation Library (GROWL), a collaborative project (JISC VRE programme) between CCLRC Daresbury Laboratory and the Universities of Cambridge and Lancaster. http://www.growl.org.uk/

[26] The Large Hadron Collider. http://lhc.web.cern.ch

[27] The LHC Computing Grid Project. http://lcg.web.cern.ch/LCG

[28] Software for Analysis of Binary Recurrent Events (SABRE). http://sabre.lancs.ac.uk/

[29] CERN press release PR17.06, "World's largest superconducting magnet switches on", 20.11.2006 http://press.web.cern.ch/Press/PressReleases/Releases2006/PR 17.06E.html 